\title{
Buckling Analysis of a Rotationally Restrained Single Walled Carbon Nanotube Embedded In An Elastic Medium Using Nonlocal Elasticity
}

\author{
Mustafa Özgür YAYLI ${ }^{a}$ \\ ${ }^{a}$ Uludağ University Engineering Faculty Department of Civil Engineering, Bursa,Turkey \\ *E-mail address: ozguryayli@uludag.edu.tr \\ Received date: July 2016 \\ Accepted date: August 2016
}

\begin{abstract}
The buckling analysis of a simply supported single walled carbon nanotube embedded in an elastic medium with rotationally restrained boundary conditions is presented via nonlocal elasticity theory. A Fourier sine series incorporated with Stokes' transformation is employed for the simulation of single walled carbon nanotube deflections. The Fourier coefficients for a embedded carbon nanotube having ends with rotational restraints are obtained by the substitution of deflection function and its derivatives into the governing differential equation. The explicit expressions are derived for the critical buckling loads by using nonlocal boundary conditions in terms of rotational restraint parameters. A detailed parametric investigation have been carried out to study the effects of the rotational spring parameters on the size-dependent stability characteristics of the carbon nanotube.
\end{abstract}

Keywords: Buckling analysis, carbon nanotube, Fourier sine series, Stokes' transformation, elastic medium

\section{Introduction}

Atomistic simulations and experimental findings have proved a significant small size effect in the mechanic performance of material at micro and nano scale [1-3]. The application of classical elasticity theories might be questionable in the theoretical analysis of nanosized structures, since classical continuum theories lack the accountability of the small size effects. Carbon nanotubes (nanobeams) have many potential applications, such as graphene transistors, solar cells, gas detection, diagnosis devices, ultra capacitors and ultra strength composite materials [4-5]. In addition, they are ultra light and are highly sensitive to its environment changes [6].

In the design of carbon nanotubes, it is of importance to acquire the real mechanical behaviors of nanotubes accurately. The application of classic continuum theory may be questionable in the mechanical analysis of carbon nanotubes. Classical continuum theory (classical elasticity theory) is material scale-free theory and this theory lacks the accountability of the small size effects. In order to overcome the shortcomings of classical continuum theory (classical elasticity theory), different formats of higher order continuum theories such as micro-polar elasticity theory [7-9], nonlocal elasticity theory [10], couple stress theory [11] and the modified couple stress approach [12,13] have been receiving much attention in investigating micro/nano structures. Since experiments including small size effects are expensive, the definition of appropriate physical models for nanobeams (carbon nanotubes) is a crucial issue concerning the theoretical and mathematical framework of nanosized structures. 
Eringen [14] proposed the nonlocal elasticity theory in 1970s. to overcome the shortcomings of classical elasticity models. This size dependent model (nonlocal elasticity theory) states that the stress field at a point not only depends on the strain field at that point but also on strains at all other points of the body. Many research works correlated to this nonlocal model have been so far explored to analyze the mechanical behavior of nanosized structures, see Refs. [15-24].

In this work, on the basis of the nonlocal elasticity theory, a new unified model is presented for the buckling analysis of single-walled carbon nanotubes embedded in an elastic medium with rotational restraints. The lateral displacement function is sought as the superposition of a Fourier sine series and Stokes' transformation that is used to take care of the deformable boundary conditions (rotational restraints). Present eigenvalue algorithm can be degenerate to the rigid supporting nanostructure (simply supported, clamped-pinned, clamped-clamped) in the cases of assigning the proper values of rotational restraints. The remarkable convergence of the present solution have been repeatedly demonstrated through the numerical examples.

\section{Formulation of the problem}

According to nonlocal elasticity proposed by Eringen the constitutive equation is represented by the following relation

$$
\left(1-\mu \nabla^{2}\right) \sigma^{n l}=\sigma^{l}
$$

where $\mu$ is the nonlocal parameter, $\nabla^{2}$ is the Laplacian operator, $\sigma^{n l}$ is the nonlocal stress tensor. $\sigma^{l}$ denotes the local tensor related to strain

$$
\sigma^{l}(x)=\kappa(x): \varepsilon(x)
$$

where $\kappa(x)$ is the fourth order tensor of elasticity. The ':' symbol denotes the double dot product. The following equilibrium equations in terms of the lateral deflections can be written

$$
\begin{gathered}
\frac{d V}{d x}=k_{w} w, \\
V-\frac{d M}{d x}+p \frac{d w}{d x}=0,
\end{gathered}
$$

where $p$ is the in-plane axial load. $k_{w}$ represents the constant of the foundation, known as Winkler's constant, $w$ denotes the lateral displacement of the carbon nanotube. The constitutive relation in nonlocal elasticity is given by

$$
M-\mu \frac{d^{2} M}{d x^{2}}=-E I \frac{d^{2} w}{d x^{2}} .
$$

Consequently substituting the Eqs. (1) and (2) in shear force and bending moment is written as 


$$
\begin{gathered}
V=-E I \frac{d^{3} w}{d x^{3}}+\mu\left[p \frac{d^{3} w}{d x^{3}}+k \frac{d w}{d x}\right]-p \frac{d w}{d x}, \\
M=-E I \frac{d^{2} w}{d x^{2}}+\mu\left[p \frac{d^{2} w}{d x^{2}}+k_{w} w\right]
\end{gathered}
$$

Further considering Eqs (6) and (7), the fourth order governing differential equation of a single walled carbon nanotube embedded in elastic medium is given by $[25,26]$

$$
E I \frac{d^{4} w}{d x^{4}}+p \frac{d^{2} w}{d x^{2}}-\mu k \frac{d^{2} w}{d x^{2}}-\mu p \frac{d^{4} w}{d x^{4}}+k_{w} w=0
$$

In the case of the deformable boundary conditions, the analytical solution of Eq. (8) is difficult to obtain, so Fourier series expansion together with Stokes transformation will be adopted in this work for the solution of Eq. (8). The displacement function $w(x)$ is described in three separate regions, two for boundary points and the other for the intermediate places between the simply supported ends with rotational restraints:

$$
\begin{array}{cc}
w(x)=\xi_{0}, & x=0, \\
w(x)=\xi_{L}, & x=L, \\
w(x)=\sum_{m=0}^{\infty} T_{m} \sin \left(\alpha_{m} x\right) & 0<x<L,
\end{array}
$$

where

$$
\alpha_{m}=\frac{m \pi}{L}
$$

The coefficient $\left(T_{m}\right)$ in Eq. (11) can be written as;

$$
T_{m}=\frac{2}{L} \int_{0}^{L} w(x) \sin \left(\alpha_{m} x\right) d x
$$

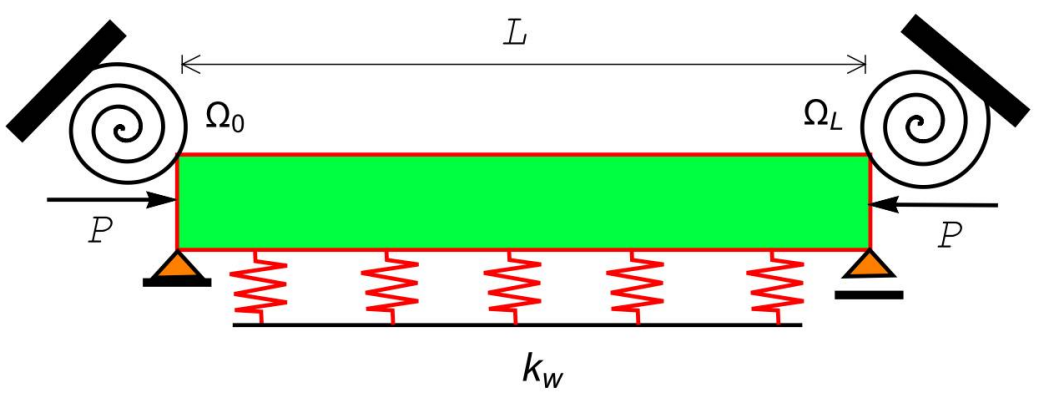

Figure 1: A simply supported carbon nanotube embedded in elastic medium with rotational restraints 
The first derivative of Eq. (11) yields

$$
w^{\prime}(x)=\sum_{m=1}^{\infty} \alpha_{m} T_{m} \cos \left(\alpha_{m} x\right)
$$

Eq. (14) can be defined as a Fourier cosine series:

$$
w^{\prime}(x)=\frac{f_{0}}{L}+\sum_{m=1}^{\infty} f_{m} \cos \left(\alpha_{m} x\right)
$$

The coefficients $\left(f_{0}, f_{m}\right)$ in Eq. (15) are given by

$$
\begin{gathered}
f_{0}=\frac{2}{L} \int_{0}^{L} w^{\prime}(x) d x=\frac{2}{L}[w(L)-w(0)], \\
f_{m}=\frac{2}{L} \int_{0}^{L} w^{\prime}(x) \cos \left(\alpha_{m} x\right) d x \quad(m=1,2 \ldots) .
\end{gathered}
$$

Using integration by parts, we get;

$$
\begin{gathered}
f_{m}=\frac{2}{L}\left[w(x) \cos \left(\alpha_{m} x\right)\right]_{0}^{L}+\frac{2}{L}\left[\alpha_{m} \int_{0}^{L} w(x) \sin \left(\alpha_{m} x\right) d x\right] \\
f_{m}=\frac{2}{L}\left[(-1)^{m} w(L)-w(0)\right]+\alpha_{m} T_{m} .
\end{gathered}
$$

The above transformation procedure is known as Stokes' transformation. Then, the first derivative of lateral deflection function is obtained as follows:

$$
\frac{d w(x)}{d x}=\frac{\xi_{L}-\xi_{0}}{L}+\sum_{m=1}^{\infty} \cos \left(\alpha_{m} x\right)\left(\frac{2\left((-1)^{m} \xi_{L}-\xi_{0}\right)}{L}+\alpha_{m} T_{m}\right)
$$

The higher order derivatives of lateral deflection function $w(x)$ can be separately obtained by employing Stokes' transformation as follows [24]:

$$
\begin{gathered}
\frac{d^{2} w(x)}{d x^{2}}=-\sum_{m=1}^{\infty} \alpha_{m} \sin \left(\alpha_{m} x\right)\left(\frac{2\left((-1)^{m} \xi_{L}-\xi_{0}\right)}{L}+\alpha_{m} T_{m}\right), \\
\frac{d^{3} w(x)}{d x^{3}}=\frac{\xi_{L} "-\xi_{0}^{\prime \prime}}{L}+\sum_{m=1}^{\infty} \cos \left(\alpha_{m} x\right)\left(\frac{2\left((-1)^{m} \xi_{L}^{\prime \prime}-\xi_{0} "\right)}{L}-\alpha_{m}^{2}\left(\frac{2\left((-1)^{m} \xi_{L}-\xi_{0}\right)}{L}+\alpha_{m} T_{m}\right)\right),
\end{gathered}
$$




$$
\frac{d^{4} w(x)}{d x^{4}}=-\sum_{m=1}^{\infty} \alpha_{m} \sin \left(\alpha_{m} x\right)\left(\frac{2\left((-1)^{m} \xi_{L} "-\xi_{0} "\right)}{L}-\alpha_{m}^{2}\left(\frac{2\left((-1)^{m} \xi_{L}-\xi_{0}\right)}{L}+\alpha_{m} T_{m}\right)\right) .
$$

The coefficient $\left(T_{m}\right)$ in Eq. (14) can be calculated from Eq. (8) in terms of $\xi_{0}, \xi_{L}, \xi_{0}$ " and $\xi_{L}$ " as follows:

$$
T_{m}=\frac{2 \alpha_{m}\left(\overline{P_{k_{w}, \mu}}\left(-\xi_{0}+(-1)^{m} \xi_{L}\right)+\overline{P_{E I, \mu}}\left(\alpha_{m}^{2}\left(\xi_{0}+(-1)^{1+m} \xi_{L}\right)-\xi_{0}{ }^{\prime \prime}+(-1)^{m} \xi_{L} "\right)\right.}{L\left(k_{w}+\overline{P_{k_{w}, \mu}} \alpha_{m}^{2}+\overline{P_{E I, \mu}} \alpha_{m}^{4}\right)}
$$

where

$$
\begin{aligned}
& \overline{P_{k_{w}, \mu}}=k_{w} \mu-P, \\
& \overline{P_{E I, \mu}}=E I-P \mu .
\end{aligned}
$$

The lateral deflection function $w(x)$ for the buckling of a single walled carbon nanotube having no restraints and supports becomes

$$
w(x)=\sum_{m=1}^{\infty} \frac{2 \alpha_{m}\left(\overline{P_{k_{w}, \mu}}\left(-\xi_{0}+(-1)^{m} \xi_{L}\right)+\overline{P_{E I, \mu}}\left(\alpha_{m}^{2}\left(\xi_{0}+(-1)^{1+m} \xi_{L}\right)-\xi_{0} "+(-1)^{m} \xi_{L} "\right)\right.}{L\left(k_{w}+\overline{P_{k_{w}, \mu}} \alpha_{m}^{2}+\overline{P_{E I, \mu}} \alpha_{m}^{4}\right)} \sin \left(\alpha_{m} x\right) .
$$

While solving the governing differential equation with rigid boundary conditions researchers preferred differential transform method like in [26]. In this work, an attempt is made to propose a new analytic approach for buckling analysis of a carbon nanotube embedded in an elastic medium with deformable boundary conditions.

\section{Boundary conditions}

Consider a embedded single walled carbon nanotube with rotational restraints at both simply supported ends (see Figure 1). This model bridges the gap between simply supported and the clamped boundary conditions, which is of great significance for the application of the continuum approach to carbon nanostructures. The following deformable boundary conditions are used,

$$
\begin{array}{lll}
\xi_{0}=0 & \Omega_{0} \frac{\partial w(x)}{\partial x}=E I \xi_{0} " & x=0, \\
\xi_{L}=0 & \Omega_{L} \frac{\partial w(x)}{\partial x}=E I \xi_{L} " & x=L,
\end{array}
$$

After putting the Eqs. (20) and (24) into Eqs. (28)-(29), one obtains the following two homogeneous equations 


$$
\begin{aligned}
& \left\{-1+\sum_{m=1}^{\infty} \frac{2(\mathrm{EI}-\mathrm{P} \mu) \Omega_{0} L m^{2} \pi^{2}}{-\mathrm{L}^{2} \mathrm{~m}^{2} \mathrm{P} \pi^{2}+\mathrm{m}^{4} \pi^{2}(\mathrm{EI}-\mathrm{P} \mu)+\mathrm{k}_{w}\left(\mathrm{~L}^{4}+\mathrm{L}^{2} \mathrm{~m}^{2} \pi^{2} \mu\right)}\right\} \xi_{0} " \\
& -\left\{\sum_{m=1}^{\infty} \frac{2(\mathrm{EI}-\mathrm{P} \mu)(-1)^{m} \Omega_{0} L m^{2} \pi^{2}}{-\mathrm{L}^{2} \mathrm{~m}^{2} \mathrm{P} \pi^{2}+\mathrm{m}^{4} \pi^{2}(\mathrm{EI}-\mathrm{P} \mu)+\mathrm{k}_{w}\left(\mathrm{~L}^{4}+\mathrm{L}^{2} \mathrm{~m}^{2} \pi^{2} \mu\right)}\right\} \xi_{L} "=0, \\
& \left\{-\sum_{m=1}^{\infty} \frac{2(\mathrm{EI}-\mathrm{P} \mu)(-1)^{m} \Omega_{L} L m^{2} \pi^{2}}{-\mathrm{L}^{2} \mathrm{~m}^{2} \mathrm{P} \pi^{2}+\mathrm{m}^{4} \pi^{2}(\mathrm{EI}-\mathrm{P} \mu)+\mathrm{k}_{w}\left(\mathrm{~L}^{4}+\mathrm{L}^{2} \mathrm{~m}^{2} \pi^{2} \mu\right)}\right\} \xi_{0} " \\
& +\left\{-1+\sum_{m=1}^{\infty} \frac{2(\mathrm{EI}-\mathrm{P} \mu) \Omega_{L} L m^{2} \pi^{2}}{-\mathrm{L}^{2} \mathrm{~m}^{2} \mathrm{P} \pi^{2}+\mathrm{m}^{4} \pi^{2}(\mathrm{EI}-\mathrm{P} \mu)+\mathrm{k}_{w}\left(\mathrm{~L}^{4}+\mathrm{L}^{2} \mathrm{~m}^{2} \pi^{2} \mu\right)}\right\} \xi_{L} "=0 .
\end{aligned}
$$

Eqs. (30)-(31) can be written as a matrix form

$$
\left[\begin{array}{ll}
\psi_{11} & \psi_{12} \\
\psi_{21} & \psi_{22}
\end{array}\right]\left[\begin{array}{l}
\xi_{0} " \\
\xi_{L}^{\prime \prime}
\end{array}\right]=0
$$

where

$$
\begin{aligned}
& \psi_{11}=-1+\sum_{m=1}^{\infty} \frac{2(\mathrm{EI}-\mathrm{P} \mu) \Omega_{0} L m^{2} \pi^{2}}{-\mathrm{L}^{2} \mathrm{~m}^{2} \mathrm{P} \pi^{2}+\mathrm{m}^{4} \pi^{2}(\mathrm{EI}-\mathrm{P} \mu)+\mathrm{k}_{w}\left(\mathrm{~L}^{4}+\mathrm{L}^{2} \mathrm{~m}^{2} \pi^{2} \mu\right)}, \\
& \psi_{12}=-\sum_{m=1}^{\infty} \frac{2(\mathrm{EI}-\mathrm{P} \mu)(-1)^{m} \Omega_{0} L m^{2} \pi^{2}}{\mathrm{~L}^{2} \mathrm{~m}^{2} \mathrm{P} \pi^{2}+\mathrm{m}^{4} \pi^{2}(\mathrm{EI}-\mathrm{P} \mu)+\mathrm{k}_{w}\left(\mathrm{~L}^{4}+\mathrm{L}^{2} \mathrm{~m}^{2} \pi^{2} \mu\right)}, \\
& \psi_{21}=-\sum_{m=1}^{\infty} \frac{2(\mathrm{EI}-\mathrm{P} \mu)(-1)^{m} \Omega_{L} L m^{2} \pi^{2}}{\mathrm{~L}^{2} \mathrm{~m}^{2} \mathrm{P} \pi^{2}+\mathrm{m}^{4} \pi^{2}(\mathrm{EI}-\mathrm{P} \mu)+\mathrm{k}_{w}\left(\mathrm{~L}^{4}+\mathrm{L}^{2} \mathrm{~m}^{2} \pi^{2} \mu\right)}, \\
& \psi_{22}=-1+\sum_{m=1}^{\infty} \frac{2(\mathrm{EI}-\mathrm{P} \mu) \Omega_{L} L m^{2} \pi^{2}}{-\mathrm{L}^{2} \mathrm{~m}^{2} \mathrm{P} \pi^{2}+\mathrm{m}^{4} \pi^{2}(\mathrm{EI}-\mathrm{P} \mu)+\mathrm{k}_{w}\left(\mathrm{~L}^{4}+\mathrm{L}^{2} \mathrm{~m}^{2} \pi^{2} \mu\right)} .
\end{aligned}
$$

Eq. (32) is a eigenvalue problem. The critical buckling loads can be calculated by setting the determinant of the coefficient matrix to zero

$$
\left|\begin{array}{ll}
\psi_{11} & \psi_{12} \\
\psi_{21} & \psi_{22}
\end{array}\right|=0
$$

The characteristic equation can be achieved by assigning the proper values of $\left(\Omega_{0}\right)$ and $\left(\Omega_{L}\right)$ corresponding to the restrained boundary condition.

\section{Numerical results}

In this section, the embedded single walled carbon nanotube with rotational restraints for buckling is explored based on the nonlocal approach. With the theoretical formulation proposed in this study, different numerical examples are solved in this part. Firstly, accuracy and validity of the present mathematical approach is validated. Then, the effects of rotational spring and nonlocal parameters on 
the buckling characteristics of single walled carbon nanotubes with rotational restraints under axial loading are investigated.

\subsection{Validity and convergence for buckling}

Prior to presentation of numerical results of the critical buckling loads, let us examine the accuracy of the method suggested here when applied to three special case of the physical model presented in this study, in which there is no elastic foundation. Theoretically, there are infinite terms of Fourier sine series. However, in the practical calculation, only finite terms are taken into account and the Fourier sine series should be truncated. Therefore, a convergence check study is of great importance in checking the convergence of the expansion sine series. Table 1, 2 and 3 list the critical buckling loads for the nanotubes under simply supported, clamped-clamped and clamped-hinged boundary conditions by using Fourier series expansion (FSE). The calculated critical buckling loads are computed using 150 terms of infinite series. The critical buckling loads given by Senthilkumar et al. (2010) and Pradhan and Reddy (2011) are also tabulated for direct comparison. The present results agree very well with the solutions by Senthilkumar et al. (2010) and Pradhan and Reddy (2011) in which differential transform method (DTM) applied for various length and nonlocal parameter of nanotube. According to Tables 1, 2 and 3 present results are convergent. From these tables it is clearly seen that 150 terms of infinite series are sufficient to calculate the accurate results for the present analysis.

Table 1. Verification of the proposed method for a simply supported carbon nanotub

\begin{tabular}{|c|c|c|c|c|c|c|c|c|c|}
\hline \multirow[b]{2}{*}{ Length (nm) } & \multicolumn{3}{|l|}{$\begin{array}{l}\mathrm{P}(\mathrm{nN}) \\
\mu=0 \mathrm{~nm}^{2}\end{array}$} & \multicolumn{3}{|l|}{$\begin{array}{l}\mathrm{P}(\mathrm{nN}) \\
\mu=1 \mathrm{~nm}^{2}\end{array}$} & \multicolumn{3}{|l|}{$\begin{array}{l}\mathrm{P}(\mathrm{nN}) \\
\mu=2 \mathrm{~nm}^{2}\end{array}$} \\
\hline & $\begin{array}{l}\text { P(exact) } \\
\text { Ref.[25] }\end{array}$ & $\begin{array}{l}\text { P(DTM) } \\
\text { Ref[26] }\end{array}$ & $\begin{array}{l}\mathrm{P}(\mathrm{FSE}) \\
\text { Present }\end{array}$ & $\begin{array}{l}\mathrm{P}(\text { exact }) \\
\text { Ref.[25] }\end{array}$ & $\begin{array}{l}\text { P(DTM) } \\
\text { Ref[26] }\end{array}$ & $\begin{array}{l}\mathrm{P}(\mathrm{FSE}) \\
\text { Present }\end{array}$ & $\begin{array}{l}\text { P(exact) } \\
\text { Ref.[25] }\end{array}$ & $\begin{array}{l}\text { P(DTM) } \\
\text { Ref.[26] }\end{array}$ & $\begin{array}{l}\mathrm{P}(\mathrm{FSE}) \\
\text { Present }\end{array}$ \\
\hline 10,00 & 4.8447 & 4.8447 & 4.8447 & 4.4095 & 4.4095 & 4.4095 & 4.0460 & 4.0460 & 4.0460 \\
\hline 12,00 & 3.3644 & 3.3644 & 3.3644 & 3.1486 & 3.1486 & 3.1486 & 2.9588 & 2.9588 & 2.9588 \\
\hline 14,00 & 2.4718 & 2.4718 & 2.4718 & 2.3533 & 2.3533 & 2.3533 & 2.2456 & 2.2456 & 2.2456 \\
\hline 16,00 & 1.8925 & 1.8925 & 1.8925 & 1.8222 & 1.8222 & 1.8222 & 1.7569 & 1.7569 & 1.7569 \\
\hline 18,00 & 1.4953 & 1.4953 & 1.4953 & 1.4511 & 1.4511 & 1.4511 & 1.4094 & 1.4094 & 1.4094 \\
\hline 20,00 & 1.2112 & 1.2112 & 1.2112 & 1.1820 & 1.1820 & 1.1820 & 1.1542 & 1.1542 & 1.1542 \\
\hline
\end{tabular}

Table 2. Verification of the proposed method for a clamped-clamped carbon nanotube

\begin{tabular}{|c|c|c|c|c|c|c|c|c|c|}
\hline \multirow[b]{2}{*}{ Length (nm) } & \multicolumn{3}{|l|}{$\begin{array}{l}\mathrm{P}(\mathrm{nN}) \\
\mu=0 \mathrm{~nm}^{2}\end{array}$} & \multicolumn{3}{|l|}{$\begin{array}{l}\mathrm{P}(\mathrm{nN}) \\
\mu=1 \mathrm{~nm}^{2}\end{array}$} & \multicolumn{3}{|l|}{$\begin{array}{l}\mathrm{P}(\mathrm{nN}) \\
\mu=2 \mathrm{~nm}^{2}\end{array}$} \\
\hline & $\begin{array}{l}\mathrm{P}(\text { exact }) \\
\text { Ref.[25] }\end{array}$ & $\begin{array}{l}\text { P(DTM) } \\
\text { Ref[26] }\end{array}$ & $\begin{array}{l}\text { P(FSE) } \\
\text { Present }\end{array}$ & $\begin{array}{l}\mathrm{P}(\text { exact}) \\
\text { Ref.[25] }\end{array}$ & $\begin{array}{l}\text { P(DTM) } \\
\text { Ref[26] }\end{array}$ & $\begin{array}{l}\mathrm{P}(\mathrm{FSE}) \\
\text { Present }\end{array}$ & $\begin{array}{l}\text { P(exact) } \\
\text { Ref.[25] }\end{array}$ & $\begin{array}{l}\text { P(DTM) } \\
\text { Ref.[26] }\end{array}$ & $\begin{array}{l}\mathrm{P}(\mathrm{FSE}) \\
\text { Present }\end{array}$ \\
\hline 10,00 & 19.379 & 19.379 & 19.379 & 13.8939 & 13.8939 & 13.8939 & 10.8280 & 10.8280 & 10.8280 \\
\hline 12,00 & 13.458 & 13.458 & 13.458 & 10.6520 & 10.6520 & 10.6520 & 8.6917 & 8.6917 & 8.6917 \\
\hline
\end{tabular}




\begin{tabular}{|l|l|l|l|l|l|l|l|l|l|}
14,00 & 9.877 & 9.877 & 9.877 & 8.2296 & 8.2296 & 8.2296 & 7.0479 & 7.0479 & 7.0479 \\
\hline 16,00 & 7.469 & 7.469 & 7.569 & 6.5585 & 6.5585 & 6.5585 & 5.7854 & 5.7854 & 5.7854 \\
\hline 18,00 & 5.981 & 5.981 & 5.981 & 5.3375 & 5.3375 & 5.3375 & 4.8091 & 4.8091 & 4.8091 \\
\hline 20,00 & 4.845 & 4.845 & 4.844 & 4.4095 & 4.4095 & 4.4095 & 4.0460 & 4.0460 & 4.0460 \\
\hline
\end{tabular}

Table 3. Verification of the proposed method for a clamped-hinged carbon nanotube

\begin{tabular}{|c|c|c|c|c|c|c|c|c|c|}
\hline \multirow[b]{2}{*}{ Length (nm) } & \multicolumn{3}{|l|}{$\begin{array}{l}\mathrm{P}(\mathrm{nN}) \\
\mu=0 \mathrm{~nm}^{2}\end{array}$} & \multicolumn{3}{|l|}{$\begin{array}{l}\mathrm{P}(\mathrm{nN}) \\
\mu=1 \mathrm{~nm}^{2}\end{array}$} & \multicolumn{3}{|l|}{$\begin{array}{l}\mathrm{P}(\mathrm{nN}) \\
\mu=2 \mathrm{~nm}^{2}\end{array}$} \\
\hline & $\begin{array}{l}\text { P(exact) } \\
\text { Ref.[25] }\end{array}$ & $\begin{array}{l}\text { P(DTM) } \\
\text { Ref[26] }\end{array}$ & $\begin{array}{l}\text { P(FSE) } \\
\text { Present }\end{array}$ & $\begin{array}{l}\text { P(exact) } \\
\text { Ref.[25] }\end{array}$ & $\begin{array}{l}\text { P(DTM) } \\
\text { Ref[26] }\end{array}$ & $\begin{array}{l}\text { P(FSE) } \\
\text { Present }\end{array}$ & $\begin{array}{l}\text { P(exact) } \\
\text { Ref.[25] }\end{array}$ & $\begin{array}{l}\text { P(DTM) } \\
\text { Ref.[26] }\end{array}$ & $\begin{array}{l}\text { P(FSE) } \\
\text { Present }\end{array}$ \\
\hline 10,00 & 9.8870 & 9.8870 & 9.9379 & 8.2295 & 8.2295 & 8.2925 & 7.0480 & 7.0480 & 7.1021 \\
\hline 12,00 & 6.8860 & 6.8860 & 6.9014 & 6.0235 & 6.0235 & 6.0721 & 5.3651 & 5.3651 & 5.4035 \\
\hline 14,00 & 5.0440 & 5.0440 & 5.0704 & 4.5744 & 4.5744 & 4.6125 & 4.1844 & 4.1844 & 4.2163 \\
\hline 16,00 & 3.8621 & 3.8621 & 3.8820 & 3.5804 & 3.5804 & 3.6109 & 3.3370 & 3.3370 & 3.3635 \\
\hline 18,00 & 3.0516 & 3.0516 & 3.0673 & 2.8730 & 2.8730 & 2.8978 & 2.7141 & 2.7141 & 2.7363 \\
\hline 20,00 & 2.4718 & 2.4718 & 2.4844 & 2.3533 & 2.3533 & 2.3739 & 2.2456 & 2.2456 & 2.2644 \\
\hline
\end{tabular}

It is observed from the Figure 2-a and Figure 3-a that when the stiffnesses of rotational springs are almost zero the results are exactly match with those reported by Senthilkumar et al. (2010) and Pradhan and Reddy (2011) (simply supported case). The rotational spring constants, $\left(\Omega_{0}, \Omega_{L}\right)$, are representative of how stiff the rotational spring are. Stiffer (more difficult to rotate) springs have higher spring constants $\left(\Omega_{0}=\Omega_{L}=\infty\right)$. It can be seen from the Figure 2-b and Figure 3-b that when the stiffnesses of rotational springs are large the results are match with those reported by Senthilkumar et al. (2010) and Pradhan and Reddy (2011) (clamped-clamped case). It is also seen Figure 2 and 3 that the small scale effects are more significant for large $\mu$ values when compared with small ones.

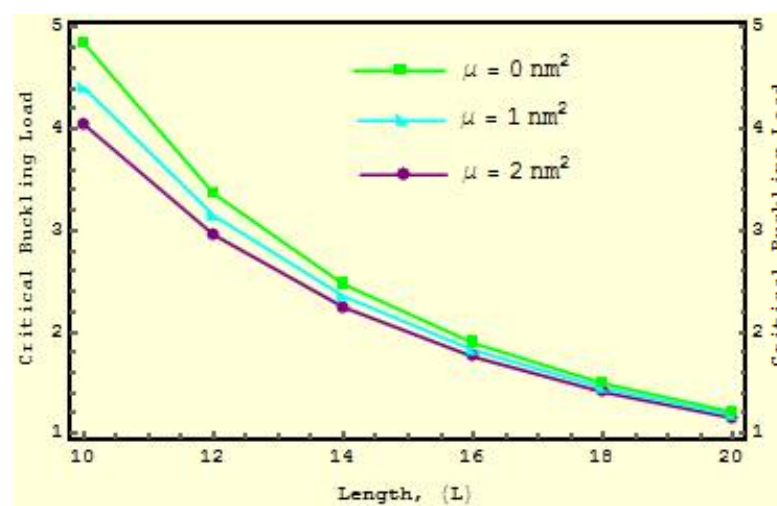

a) $k_{w}=0, \Omega_{0}=\Omega_{L}=0$

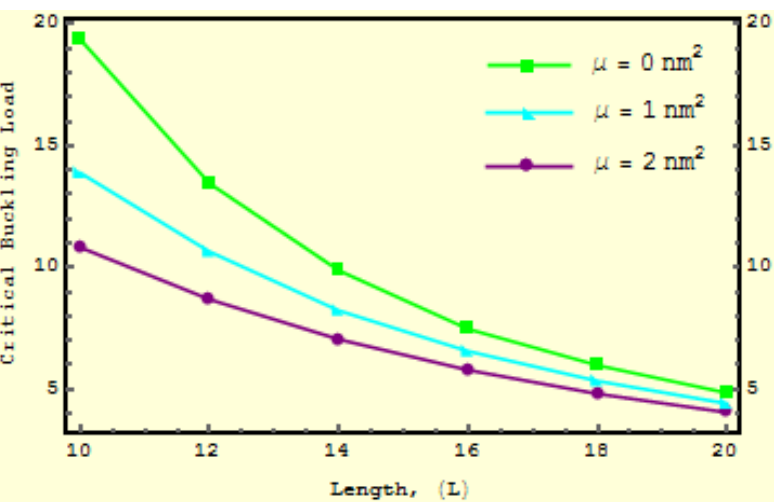

b) $k_{w}=0, \Omega_{0}=\Omega_{0}=\infty$

Figure 2: Nonlocal effect on the critical buckling loads for different nonlocal parameter 


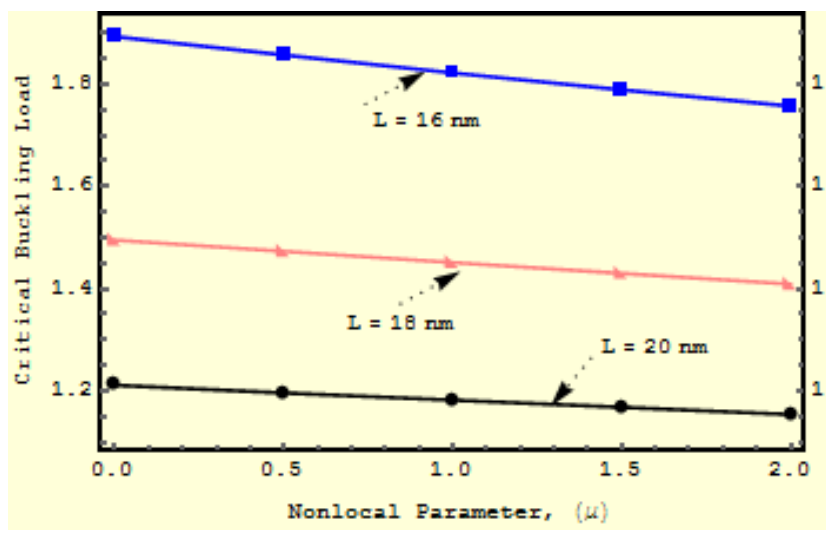

a) $k_{w}=0, \Omega_{0}=\Omega_{0}=0$

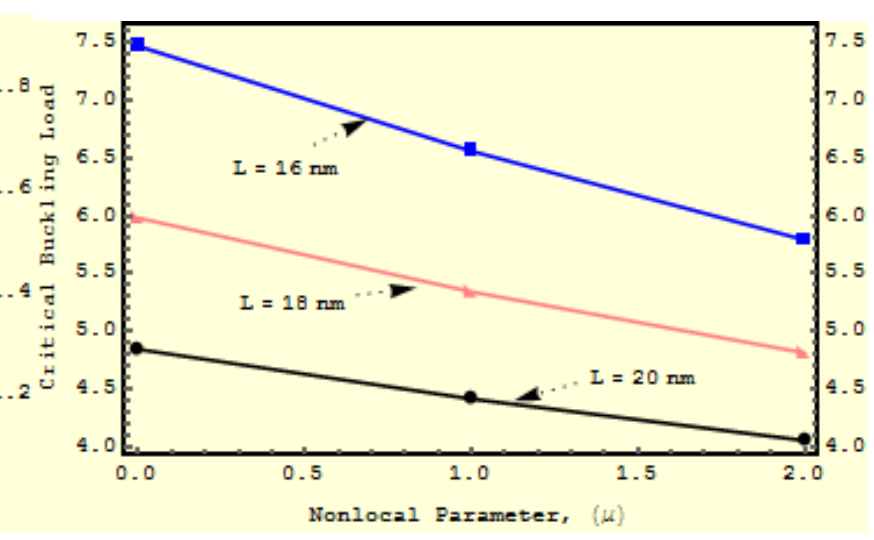

b) $k_{w}=0, \Omega_{0}=\Omega_{0}=\infty$

Figure 3: Nonlocal effect on the critical buckling loads for different length

\subsection{Effects of the rotational restraints}

To investigate the effects of the rotational restraints on the critical buckling loads, variation of critical buckling loads with different nonlocal patameters are schematically plotted in Figs. 4 and 5. Based on the results in Figs. 4 and 5, the increasing value of the rotational restraint parameter leads to an increase in the magnitude of critical buckling load. As expected, the stiffening effect of rotational restraint parameter is to increase the critical buckling load. It is also said that the presence of elastic medium enhances the rigidity of the carbon nanotube and increases the critical buckling parameter of nanotube. Using the coefficient matrix obtained in this work, the critical buckling loads of a embedded single walled carbon nanotube with different boundary conditions can be easily computed. This matrix including rotational restraint parameters can be useful in theoretical investigation, that leads to determinant calculation of a $2 \times 2$ matrix.

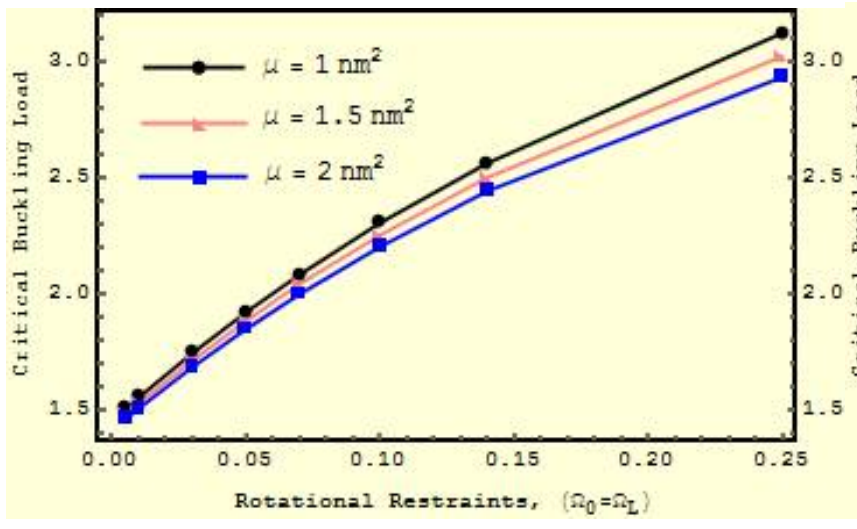

a) $k_{w}=0$

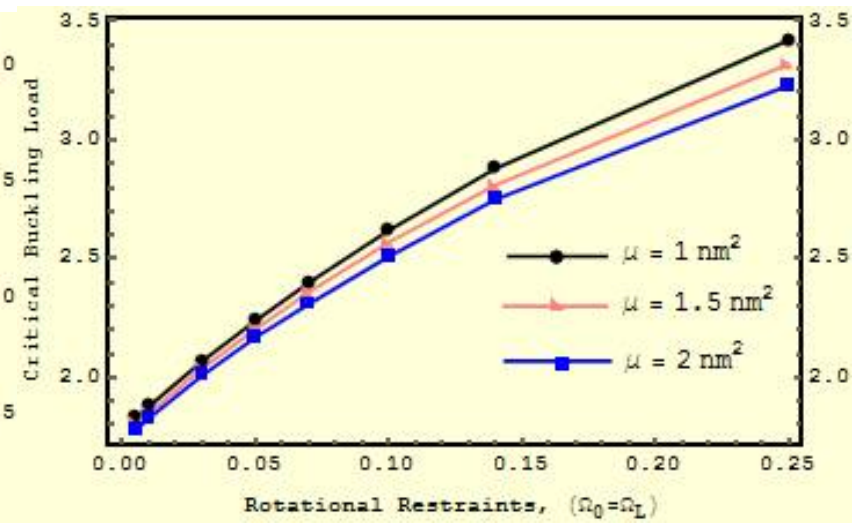

b) $k_{w}=0.01 \mathrm{nN} / \mathrm{nm}^{2} / \mathrm{nm}$

Figure 4: Effect of rotational restraints on the critical buckling loads for $\mathrm{L}=18 \mathrm{~nm}$ 


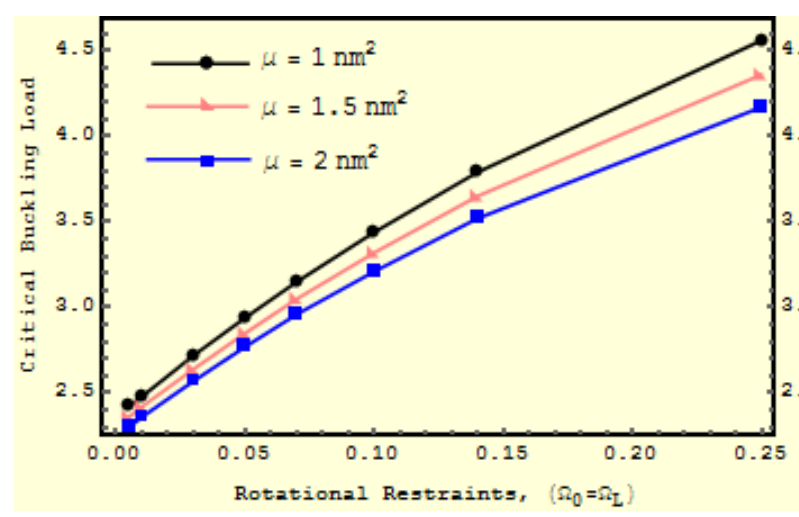

a) $k_{w}=0$

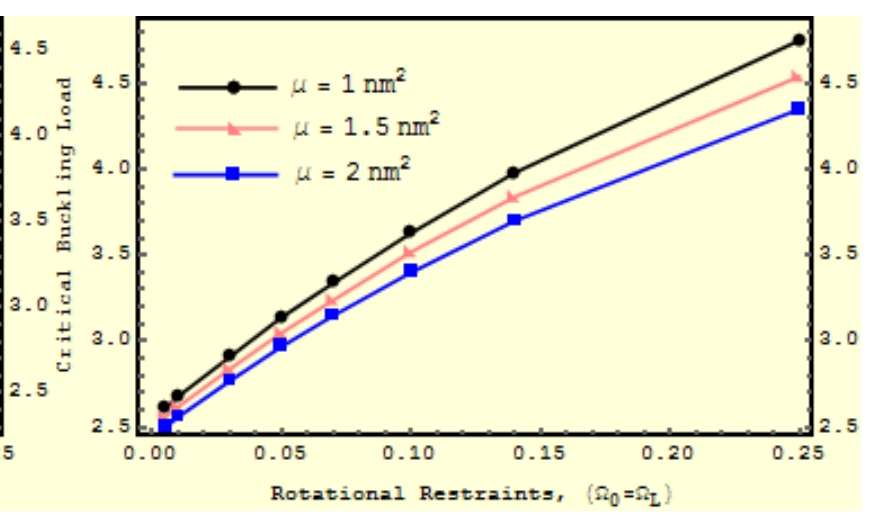

b) $k_{w}=0.01 \mathrm{nN} / \mathrm{nm}^{2} / \mathrm{nm}$

Figure 5: Effect of rotational restraints on the critical buckling loads for $\mathrm{L}=14 \mathrm{~nm}$

\section{Conclusions}

In present work, the buckling analysis of elastically restrained embedded single walled carbon nanotube under axial compression load is researched. Winkler elastic foundation approach is used to simulate the interaction between single walled carbon nanotube and elastic medium. The effects of axial compression load, small size and surrounding elastic medium are taken into account at the same time. A coefficient matrix including rotational restraint parameters is obtained with the aid of applying Stokes' transformation to corresponding nonlocal boundary conditions. Numerical studies are performed to indicate influences of material scale, spring and Winkler parameters on the critical buckling loads.

\section{References}

[1] Li, C. Y., Chou T. W., A structural mechanics approach for the analysis of carbon nanotubes, Int. J. Solids Struct. 40, 2487-2499, 2003.

[2] Chowdhury, R., Adhikari, S., Wang, C. W., Scarpa, F., A molecular mechanics approach for the vibration of single walled carbon nanotubes. Comput. Mater. Sci., 48, 730-735, 2010.

[3] Poncharal, P., Wang, Z. L., Ugarte, D., Heer, W. A. D., Electrostatic deflections and electromechanical resonances of carbon nanotubes, Science, 283, 1513-1516, 1999.

[4] Kim, P., Lieber, C., M., Nanotube Nanotweezers. Science, 286, 2148-2150, 1999.

[5] Bachtold, A., Hadley, P., Nakanihi, T., Dekker, C., Logic circuits with carbon nanotube transistors. Science, 294, 1317-1320, 2001.

[6] Wong, E. W., Sheehan, P. E., Lieber C. M., Nanobeam mechanics: elasticity, strength and toughness of nanorods and nanotubes. Science, 277, 1971-1975, 1997.

[7] Eringen, A. C., Suhubi, E. S., Nonlinear theory of simple micro-elastic solids-I. International Journal of Engineering Science. 2, 189-203, 1964.

[8] Chen, Y., Lee, J. D., Eskandarian, A., Atomistic viewpoint of the applicability of microcontinuum theories, Int J Solids Struct. 41, 2085-2097, 2004.

[9] Ramezani, S., Naghdabadi, R., Sohrabpour, S., Analysis of micropolar elastic beams, European Journal of Mechanics-A/Solids, 28, (2) 202-208, 2009.

[10] Eringen, A. C., On differential equations of nonlocal elasticity and solutions of screw dislocation and surface waves. Journal of Applied Physics, 54, 4703-4710, 1983.

[11] Toupin, R. A., Elastic materials with couple-stresses, Archive for Rational Mechanics and Analysis, 11, 385-41, 1962. 
[12] Park, S. K., Gao, X. L., Bernoulli-Euler beam model based on a modified couple stress theory. Journal of Micromechanics and Microengineering 16, (11), 2355-2359, 2006.

[13] Ma, H. M., Gao, X. L., Reddy, J. N., A microstructure-dependent Timoshenko beam model based on a modified couple stress theory. Journal of the Mechanics and Physics of Solids 56: (12) 3379-3391. 2008.

[14] Eringen, A. C., Nonlocal polar elastic continua. International Journal of Engineering Science, 10, 1-16, 1972.

[15] Civalek, Ö., Akgöz, B., Free vibration analysis of microtubules as cytoskeleton components: nonlocal Euler-Bernoulli beam modeling, Sci. Iranica Trans. B: Mech. Eng., 17, 367-375, 2010.

[16] Civalek, Ö., Demir, Ç., Bending analysis of microtubules using nonlocal Euler-Bernoulli beam theory, Appl. Math. Model., 35, 2053-2067, 2011.

[17] Rahmani, O., Pedram, O., Analysis and modeling the size effect on vibration of functionally graded nanobeams based on nonlocal Timoshenko beam theory, Int. J. Eng. Sci, 77, 55-70, 2014. [18] Wang, C.M., Kitipornchai, S., Lim, C.W., Eisenberger, M., Beam bending solutions based on nonlocal Timoshenko beam theory, J. Eng. Mech., 134, 475-481, 2008.

[19] Lu, P., Lee, H.P., Lu, C., Zhang, P.Q., Dynamic properties of flexural beams using a nonlocal elasticity model, J. Appl. Phys., 99, 73510-73518, 2006.

[20] Eltaher, M.A., Emam, S.A., Mahmoud, F.F., Static and stability analysis of nonlocal functionally graded nanobeams. Compos. Struct, 96, 82-88, 2013.

[21] Murmu, T., Pradhan, S.C., Small-scale effect on the vibration of nonuniform nanocantilever based on nonlocal elasticity theory, Physica E, 41, 1451-1456, 2009.

[22] Setoodeh, A.R., Khosrownejad, M., Malekzadeh, P., Exact nonlocal solution for post buckling of single-walled carbon nanotubes. Physica E, 43, 1730-1737, 2011.

[23] Thai, H.T., A nonlocal beam theory for bending, buckling, and vibration of nanobeams. Int. J. Eng. Sci., 52, 56-64, 2012.

[24] Yayli, M.Ö., Buckling Analysis of a Rotationally Restrained Single Walled Carbon Nanotube, Acta Physica Polonica A, 127, 3, 678-683, 2015.

[25] Senthilkumar, V., Pradhan, S.C., Pratap, G., Small scale effect on buckling analysis of carbon nanotube with Timoshenko theory by using differential transform method, Adv Sci Lett, 3, 1-7, 2010.

[26] Pradhan, S.C., Reddy G.K., Buckling analysis of single walled carbon nanotube on Winkler foundation using nonlocal elasticity theory and DTM. Computational Materials Science, 50, 1052-1056, 2011. 